\title{
La Piedra de Novelda: una roca muy utilizada en el patrimonio arquitectónico
}

\author{
Novelda Stone: widely used within the spanish \\ architectural heritage
}

\author{
R. FORT

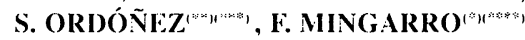 \\ ${ }^{(2)}$ Instituto de (Geología Económica. (CSIC-UCM). Facultad de Ciencias Geológicas \\ ${ }^{1-m}$ Laboratorio de Petrología Aplicada. Unidad Asociada Univ. de Alicante (CSIC) \\ "Dep. de Ciencias de la Tierra. Univ. de Alicante \\ (...rin'Dep. de Petrología y Geoquímica. Facultad de Ciencias Geológicas. Univ. Complutense de Madrid
}

Fecha de recepción: 4-XII-(0)

RESUMEN

La Piedra de Novelda es una roca carbonática del tipo biocalcarenita. Se extrae del área de Vinalopó Medio (Alicante, España oriental). La piedra de Novelda es conocida como material de construcción desde el siglo XIII. Desde finales del siglo XIX hasta la actualidad, se ha utilizado en edificios y monumentos de Madrid y Valencia, así como Alicante. En el pasado, las canteras principales donde se extrajo este tipo de piedra se localizaban en los distritos municipales de Monórar (Piedra Almorquí). Sax (Piedra Portazgo) y Elda (Piedra Bateig). Hoy en dia, la actividad principal se sitúa en las canteras de Bateig. La piedra extraída es conocida comercialmente como Piedra Bateig. Las variedades principales de esta Piedra Bateig son: Blanco, Azul, Llano y Fantasía.

Consecuencia de las diferentes propiedades y en especial de los diferentes comportamientos hídricos, la durabilidad de los distintos tipos de Piedra de Novelda y sus patologías son diferentes. La Bateig LLano y la Piedra de Portasgo sufren desagregación granular y alveolisación y su durabilidad es menor que la del resto de las rariedades. La Bateig Blanco y Bateig Azul, junto con la Piedra Almorquí, sólo en condiciones muy extremas llegan a desarrollar fisuraciones yo descamaciones.

\section{SUMMARY}

Novelda Stone is a calcareous rock, of the biocalcarenite type. It is extracted from the "Vinalopo Medio" area (Alicante, eastern Spain). Novelda stone is known as construction material from the 13th century. From final of the 19th century until the present time, it has been used in Madrid, Valencia and Alicante. In the past, the main quarries from which this type of rock was extracted were located in the municipal districts of Monowar (Almorqui Stone), Sar (Portasgo Stone) and Elda (Bateig Stone). Nowadays, the main active extraction site is situated in the Bateig Hill quarries. The rock extracted is known commercially as Bateig Stone. The main varieties of this Bateig Stone are: White, Blue, Layer and Fantasy.

Each variety of Novelda stone has different durability rate and different pathology depending on their characteristics, particularly on their hydric hehaviour patterns. Layer Bateig and Portasgo stone undergo granular disintegration and alveolization and are less durable than the rest. White and Bluc Bateig, as well as Almorqui stone, only develop fissures or scaling under extreme conditions.
PALABRAS CLAVL: petrofísica. durabilidad. piedra de construcción. piedras porosias, patrimonio arquitectónico.
KEYWORDS: petrophysics, durability, building stome, porous stome, archite'tural heritage'. 


\section{INTRODUCCIÓN}

Como Piedra de Novelda se denomina a las biocalcarenitas procedentes de la región del Vinalopó Medio (Alicante, España). Este tipo de piedra se ha utilizado, durante los últimos sicte siglos. como piedra de construcción en el patrimonio arquitectónico de la región levantina. sobre todo en el área de Novelda ( 1 y 2). Su uso se extendió, a finales del siglo XIX, a la parte central de España, siendo este hecho propiciado por la construcción, en 1858, de la línea de ferrocarril que unía las ciudades de Madrid, Zaragoza y Alicante, y con estación de tren en el pueblo de Novelda.

Gracias a su facilidad de labra, que permitía su utilización. tanto para sillería como para ornamento, y por la disponibilidad de este material. debido a la nueva vía de transporte. esta piedra ha sido utilizada en obras nuevas de la Villa de Madrid y de su Comunidad, así como en obras de restauración para sustitución de elementos deteriorados. Así se pueden destacar el Palacio de Linares (1873), el Banco de España (1891). la Estación de Ferrocarril de Príncipe Pío (1904). el Palacio de Comunicaciones (1917), el Monumento a Alfonso XII (1922) y el Edificio de la Telefónica (1929). Como piedra de sustitución se utilizó en 1925 en la Fachada de la Universidad de Alcalá (3 y 4).

Las principales canteras utilizadas para estas obras emblemáticas se localizaban en diferentes áreas. Se pueden destacar las canteras de Portazgo ( $N$ de Petrel, en el término de Sax). Bolón (al W de Elda), Bateig (entre Elda y Novelda, al oeste de Monóvar), Almorqui (en Monóvar, al sur de Casas del Señor), así como las existentes en otras localidades como Honda Alta. Terreta. etc. Las características de los materiales extraídos variaban de unas canteras a otras. Dado que los bloques de piedra de las distintas canteras eran trasladados a la estación de ferrocarril de Novelda, donde se encontraban los talleres y aserraderos de material (manufactura), todas estas rocas, independientemente de la cantera de que procedían, se consideraban como Piedra de Novelda.

Actualmente la piedra que se comercializa procede básicamente de las canteras localizadas al Oeste de la Sierra de Bateig, siendo conocida como piedra Bateig en donde la explotación se realiza con una mayor selección y control del material extraído. Se comercializan distintas variedades en la década de los noventa: Bateig Blanco, Bateig Azul, Bateig Diamante, Bateig Fantasia y Bateig Llano. Las cuatro primeras extraídas en la ladera Oeste y la última a sus pies.

Este material sigue colocándose en obras emblemáticas de la Comunidad de Madrid, como es el casso de la Catedral de la Almudena, en 1994. y en el cerramiento

\section{INTRODUCTION}

Novelda Stone is the name given to a group of biocalcarenite stones from the "Vinalopo Medion" region. This type of stone has been widely used during the last seven centuries within the architectural heritage of eastern Spain, especially the Notelda area (1 and 2). Its use extended in the late $19^{\text {th }}$ (entury to the central part of the Spain. This expansion was favoured by the building in 1858 of the first train station in the capital, managed by the company MZA, for a line that joined the cities of Madrid. Zaragosa and Alicante with a station at Novelda.

Due to the easy carring of the material and its availability, provided by the new means of transport. Novelda stone was used in new buildings in Madrid and surroundings and for the restoration and substitution of decayed materials in older buildings. Amongst other important monuments it was used in the building of: the Linares Palace (187.3), the Bank of Spain headquarters (1891), the Principe Pio train station (1904), the Central Post Office-Palacio de las Comunicaciones (1917), the Monument to Alfonso XII (1922) and the Telefonica building (1929). It was used as a substitution stone in 1925 for the façade of the University of Alcala de Henares (3 and 4).

The rock used for these outstanding monuments was extracted from quarries in diverse areas: Portago (north from Petrel in the region of Sax), Bolon (west of Elda), Bateig (located betrieen Elda and Novelda, west of Monólar), Almorqui (in Monolar, south of Casas del Señor), as well as those located in other places such as Honda Alta, Terreta, etc. Stones extracted from different quarries had different properties. However. every block of stone extracted was then carried to Novelda train station, where the workshops and manufacturing anits were located. This led to every type of stone, regardless of its quarry of origin, being called Novelda Stone.

Nowadays the stone used comes mainly from the quarries located west of the Bateig hill. The extraction process is carried out with stricter monitoring of the material than it was in the past. Different varieties have been commercialised in the decade of the nincties: White Bateig, Blue Bateig. Diamond Bateig. Fantasy Bateig and Laver Bateig. The first four are extracted from the western slope while the latter comes from the plains at the foot of the mountains.

This stone is still one of the building materials ased for important new monuments built in Madrid, such as the' Almudena Cathedral (1994) and the main entrance' to 
de la entrada principal del cementerio de la Almudena, así como en otras Comunidades Autónomas y en países de la Comunidad Europea. La producción anual es de $18.000 \mathrm{~m}^{3}$ de piedra.

El comportamiento en obra de estos materiales a corto plazo es bueno, pero para un plazo de varias decenas de años su comportamiento de durabilidad es muy irregular, de tal forma que en algunos monumentos localizados en el municipio madrileño, de edades próximas al siglo, se aprecian ya deterioros importantes cuya recuperación y restauración es de costes elevados, mientras que en otros edificios la conservación de estos materiales es bastante aceptable.

El objetivo de este trabajo es establecer la idoneidad de la Piedra Bateig para ser utilizada en obras arquitectónicas modernas o como piedra de sustitución en obras de restauración. Para cllo se comparan las características petrofísicas y petrográficas de las variedades de Piedra Bateig y de materiales de canteras antiguas, como son las de Portazgo y de Almorquí, analizando su durabilidad.

\section{METODOLOGÍA}

En 1996 se realizó un muestreo a pie de cantera de bloques característicos de las variedades con un volumen de comercialización más alto de Bateig Blanco, Azul, Llano y Fantasía. También se realizó un muestreo sistemático del frente de explotación de encro de 1997. Se extrajo un bloque de la Cantera de Almorquí y otro de la de Portazgo. De cada uno de ellos se realizó el estudio petrográfico y mineralógico. La composición mineralógica de rocas se ha determinado por difracción de rayos $X$, fue realizada con un difractómetro Philips 1840 ). Los porcentajes relativos se estimaron mediante el uso de los poderes reflectantes.

Para la caracterización petrofísica se siguieron las normas UNE (5), para los ensayos mecánicos, y las recomendaciones de la RILEM (6), para el comportamiento hídrico. Los resultados presentados son media de seis probetas, a excepción de los ensayos de saturación y de velocidad de propagación de ultrasonidos que son ocho.

Se calculó el índice de anisotropía estructural (dM\%) en base a la velocidad de los ultrasonidos en las tres direcciones del espacio (7) con el fin de evaluar la distribución espacial de algunas propiedades físicas y su variación con los procesos de alteración: the Almudena Cemetery. The stone is also in use in other parts of the country and in countries of the European Community: Annual production is $18.000 \mathrm{mi}^{\mathrm{i}}$.

Short-term performance of this stone is good, but over several decades its durability standards become irregular: Thus, while some Madrid monuments built a century ago show significant signs of decay -with the ensuing restoration costsother monuments, built around the same time, are quite well preserved.

This paper draws a comparative analysis of the petrophysical and petrographical characteristics of the different Bateig varieties and of materials from old cuarries, such as Portasego and Almoryui, suppliers for important constructions dating from the beginning of the century. Durability and performance of the stone were analysed both for the building of new architecture, and for its use as a substitution stone.

\section{METHODOLOGY}

In 1996, samples of those varieties of stone with the greatest commercial output (White, Blue, Layer and Fantasy) were taken from the quarry and from the extraction site in january 1997. A block was also extracted from the Almorqui quarry and a smaller one from the Portasgo quarry. Petrographical. mineralogical and geochemical analyses were carried out for each one of them. The mineralogical composition of stones was determined by $X$-ray: diffraction (powder method), in a Philips 1840 diffractometer: The mineral percentage was calculated by means of the intensity of the diffraction pattern.

Different guidelines were followed for different types of tests: UNE (5), for the mechanical tests, and RILEM (6) recommendations for the hydric behaviour tests. Generally, the results displayed correspond to the average values taken from sir test samples. However, for saturation tests and ultrasound propagation tests eight samples were employed.

In order to evaluate the distribution of some properties and their change with the weathering processes, the structural anisotropy index (dM \%) was calculated in function of ultrasound velocity in three directions (7): 


$$
\mathrm{dM} \%=\left(1-\frac{2 \mathrm{VL}_{1}}{\mathrm{VL}_{2}+\mathrm{VL}_{3}}\right) \times 100
$$

$\mathrm{VL}_{1}$ : Velocidad perpendicular al plano de estratificación $\mathrm{VL}_{2}$ y $\mathrm{VL}_{3}=$ Velocidad en las otras dos direcciones perpendiculares a $\mathrm{VL}_{\text {}}$.

Las medidas cromáticas se han realizado utilizando un espectrocolorímetro (Minolta CM-2002). El iluminante utilizado ha sido el estándar $D_{65}$ y con un ángulo de visión de $10^{\circ}$. El número de medidas para cada tipo de piedra ha sido de 60, con un campo de análisis en cada determinación de $50,3 \mathrm{~mm}^{2}$. Las muestras fueron secadas a unas condiciones ambientales de $T^{a}=20 \pm 1^{\circ} \partial \mathrm{C}$ y $\mathrm{Hr}=49 \pm 2 \%$. Los parámetros cromáticos utilizados han sido los pertenecientes a la escala CIELAB, 1976 (8): L** representa la luminosidad del color, variando entre cero para el negro y 100 para el blanco. El parámetro a* varía entre +60 (rojo) y -60 (verde); mientras que $b^{*}$ oscila entre +60 (amarillo) y -60 (azul). El índice de amarillo (IA) indica el grado en que la superficie de la muestra es diferente del blanco ideal en la dirección del amarillo.

Para el estudio de la durabilidad se realizaron ciclos de hielo/deshielo y humedad/sequedad, teniéndose en cuenta los estudios de durabilidad por cristalización de sales realizados anteriormente (9). El ensayo de hielo/deshielo se realizó en 45 ciclos iniciando el ciclo a $20^{\circ} \mathrm{C}$ para ir disminuyendo la temperatura a una velocidad de $0,2^{\circ} \mathrm{C}$ por minuto, hasta alcanzar los $-15^{\circ} \mathrm{C}$, donde permanece durante 3 horas para posteriormente sufrir el deshielo de forma paulatina a la misma velocidad. Cada cuatro ciclos las muestras se sumergían en agua bidestilada durante scis horas. El ensayo de humedad/sequedad consistió en la realización de 20 ciclos de inmersión en agua de las muestras durante 6 horas y posterior secado a $100^{\circ} \mathrm{C}$ durante 18 horas. Para evaluar el cfecto de ambos ensayos se controló la pérdida de peso y variación de la velocidad de propagación de ultrasonidos y su saturación en agua.

Para la caracterización del sistema poroso se determinó la distribución de tamaños de poros por medio de un porosímetro de mercurio (Autoscan-33).

\section{CARACTERÍSTICAS PETROGRÁFICAS}

La Piedra de Novelda se extrac de una unidad transgresiva del terciario (Mioceno Medio-Superior). Esta unidad es posterior a las deformaciones mayores que generaron las Cordilleras Béticass en Alicante (España). Este mioceno está ampliamente representado en la Sicrra de Bateig. Dentro de esta unidad existen variaciones litológicas laterales (cambios de facies) y verticales con características petrológicas diferentes auncue de aspecto muy similar. Su carácter transgresivo hace que alternen
$V L_{i}$ : Velocity (perpendicular to stratification plane) $V L_{2}$ and $V L_{i}=V e l o c i t y$ (other wo directions)

A spectrocolorimeter (Minolta CM-2002) was used to measure chromatic values. A standard $D_{65}$ "ras used for lighting at an angle of $10^{\prime \prime}$. For each stone variety, 60 sets of readings were taken from a sample of $50.3 \mathrm{~mm}^{2}$ for each test. Samples dried off in the following atmospheric conditions: $\mathrm{T}=20 \pm l^{\circ} \mathrm{C}$ y $\mathrm{Hr}=49 \pm 2 \%$. The chromatic parameters applied correspond to the CIELAB scale (8). $L^{*}$ represents the lightness index, whit 0 being its scale for the colour black and 100 representing white. Where $a^{*}$ is more positive the greater the colour red, where it is more negative greater the colour green. The index of yellow (IA) it indicates the degree in that the surface of the sample is different from the ideal target in the address of the yellow:

For the study of durability, freesing-thawing and wetting-dryness cycles were carried out. It has been taken into account dates of durability salt crystallisation test made in previous works (9). Freezing/thawing tests were carried out in 25 cycles, with temperature dropping from $20^{\circ} \mathrm{C}$ to $-15^{\circ} \mathrm{C}$ at a rate of $0.2{ }^{\circ} \mathrm{C}$ a minute. Samples remain at the lowest temperature for 3 hours and then the thawing process starts, at the same rate as the fiee-ing. After every four cycles, samples were submerged for six hours in bidistilled water: Humidity-dryness test consisted on the realisation of 20 immersion cycles in water of the samples during 6 hours and later drying at $100^{\circ} \mathrm{C}$ during 18 hours. In order to assess the effect of both tests, weight loss and changes in ultrasound velocity and water saturation were monitored.

Pore size distribution has been studied by mercury porosimetry using an Autoscan-33 porosimeter.

\section{PETROGRAPHICAL CHARACTERISTICS}

Novelda Stone is extracted from a Tertiary (MediumUpper Miocene) transgressive unit dating firom the period following the main transformations that led to the forming of the mountain ranges (Béticas) in the south-eastern part of Spain (Alicante). Miocene terrains are abundant in the Bateig Hills. Within the transgressive unit there are various lateral (facies changes) and vertical lithological variations, clearly differentiated petrologically but. nonetheless, very 
episodios netamente marinos con otros más continentales, para terminar con facies lacustres. Esto hace que la piedra de explotaciones antiguas, que aportaron material de sillería a monumentos emblemáticos, no tenga las características específicas correspondientes a las actuales de la Piedra Bateig.

La Piedra Bateig es una roca caliza con calcita de bajo contenido en magnesio, y en menor proporción detritos de dolomita, cuarzo, feldespato y filosilicatos (10 y 11), así como cristobalita y pirita en la variedad azul. Los filosilicatos son principalmente illitas con diferente grado de cristalinidad y en menor proporción esmectitas. La piedra Bateig petrológicamente es una roca calcárea aloquímica (biocalcarenita) con foraminíferos (Globigerinas, rotálidos, textuláridos, etc.) y restos de briozoos, moluscos y equinodermos (Tabla 1), así como de cuarzo detrítico en diferentes proporciones. La variedad Llano es la que presenta un mayor porcentaje de terrígenos que se aproximan al 30) $\%$, conservando la laminación original de deposición. Muchos cuarzos presentan inclusiones de anhidrita. El cemento de estas piedras es escaso, no superando el $10 \%$, constituido por esparíta y en menor proporción por cemento silíceo.

La piedra procedente de Portazgo presenta un porcentaje de detríticos de cuarzo y feldespatos del $15 \%$, estando los bioclastos constituidos por foraminíferos, globigerinas y rotálidos, siendo similar in appearance. Due to its transgressive nature. purely marine episodes combine with other more continental episodes, and lacustrine facies. This should explain why rock extracted in the past does not share the characteristics of the rock nowadays considered a. Bateig Stone.

Bateig Stone is a limestone variety with low-magnesium calcite and dolomite, and smaller amounts of quart:feldspar and phyllosilicate traces (10 and II), as well as crystobalite and pyrite in the Blue variety. Phyllosilicates are mainly illite with different degrees of crystallinity. There is also smectite in a smaller proportion. Petrologically, Bateig stone is an allochemical, calcareous stone (biocalcarenite) with foraminifers (globigerinid, rotaliid, textularian, etc.) and bryozoan, mollusc and echinoderm traces (Table 1), as well as fragmentary quart in different amounts. The Layer variety contains the highest percentage (around 30\%) of terrigenous materials preserving its original deposition lamination. Very often quart: contains anhydrite. There is hardly any cementing material (under 10\%) and cement is constituted by sparite and, to a smaller extent silica.

Portazgo stone contains $15 \%$ fragmentary quartz and feldspar, while bioclasts are constituted by foraminifera: globigerinids and rotaliids. Portazgo stone is classified as biomicrite and biosparite. This

TABLA $1 / T A B L E I$

Composición textural de la fracción carbonatada de diferentes variedades de la Piedra Novelda (Textural composition of the carbonated fraction of different varieties of the Novelda Stone)

\begin{tabular}{|c|c|c|c|c|}
\hline $\begin{array}{l}\text { VARIEDAD } \\
\text { Variety }\end{array}$ & $\begin{array}{c}\% \\
\text { FÓSILES } \\
\text { Fossils }\end{array}$ & $\begin{array}{l}\text { TIPOLOGÍA } \\
\text { Type }\end{array}$ & $\begin{array}{c}\% \\
\text { ORTOQUIMICOS } \\
\text { Ortochems }\end{array}$ & $\begin{array}{l}\text { TIPOLOGÍA } \\
\text { Type }\end{array}$ \\
\hline $\begin{array}{l}\text { BATEIG BLANCO } \\
\text { White Bateig }\end{array}$ & $60-70$ & $\begin{array}{l}\text { foraminíferos, briozoos, } \\
\text { pelecípodos }\end{array}$ & $30-40$ & $\begin{array}{l}\text { micrita: } 15 \%-20 \% \\
\text { esparita: } 5 \%-10 \%\end{array}$ \\
\hline $\begin{array}{l}\text { BATEIG AZUL } \\
\text { Blue Bateig }\end{array}$ & $65-75$ & $\begin{array}{l}\text { foraminíferos, briozoos, } \\
\text { equinodermos }\end{array}$ & $25-35$ & $\begin{array}{l}\text { micrita15\%-20\% } \\
\text { esparita: } 5 \%-10 \%\end{array}$ \\
\hline $\begin{array}{l}\text { BATEIG LLANO } \\
\text { Layer Bateig }\end{array}$ & $90-95$ & $\begin{array}{l}\text { foraminíferos, briozoos, } \\
\text { pelecipodos }\end{array}$ & $5-10$ & esparita $5 \%-10 \%$ \\
\hline $\begin{array}{l}\text { BATEIG FANTASIA } \\
\text { Fantasy Bateig }\end{array}$ & $65-80$ & $\begin{array}{l}\text { foraminíferos, briozoos, } \\
\text { pelecípodos }\end{array}$ & $20-35$ & $\begin{array}{l}\text { micrita y/o esparita } \\
20 \%-25 \%\end{array}$ \\
\hline ALMORQUÍ & $70-85$ & foraminíferos & $15-30$ & microesparita $25-30 \%$. \\
\hline PORTAZGO & $65-75$ & $\begin{array}{l}\text { foraminíferos, } \\
\text { equinodermos }\end{array}$ & $25-35$ & micrita $25-35 \%$ \\
\hline
\end{tabular}


clasificada como biomicrita o bioesparíta. Esta variedad de las piedras tipo Novelda es la que presenta un mayor porcentaje de filosilicatos que llegan alcanzar el 1.5 c (Tabla 2). La piedra procedente de Almorquí es la que tiene una proporción menor de terrígenos. no superando el $7 \%$ de cuarzo y feldespatos. Los restos fosilíferos estan constituidos por foraminíferos, globigerinas y restos de moluscos y briozoos, siendo clasificada como biomicrita. Puede aparecer algo de glauconita. La matriz es micrítica o microesparítica superando el $20 \%$ (Tabla 1).

\section{CARACTERÍSTICAS PETROFÍSICAS}

El color de los materiales es muy similar para todos los tipos estudiados a excepción del Bateig Azul que, a simple vista, se diferencia perfectamente por su tono más oscuro. En la Tabla 3 se muestran los parámetros cromáticos $L^{*}, a^{*}, b^{*}$ y el Indice de Amarillo (IA), así
Vovelda variety contains the highest amount of phyllosilicates, up to $15 \%$ sometimes (Table 2 ). Almorqui stone contains the lowest amount of terrigenous components, below $7 \%$ of quarts and feldspar. Fossil remains correspond to foraminifers (globigerinids) and traces of molluses and bryosoans. This stone is classed as biomicrite. Traces of glauconite may be found. The matrix is over 20\% micrite and microsparite (Table 1 ).

\section{PETROPHYSICAL CHARACTERISTICS}

Colour is quite similar in all materials studied except Blue Bateig, which, at first sight, is easily identified due to its darker hue. Chromatic parameters $L^{*}$, $a^{*}$ and $b^{*}$. the Yellow Index (YI) and Munsell nomenclature (12) are displayed on Table 3. Of all the Novelda Stone

TABLA 2/TABI.E 2

Componentes mineralógicos mayoritarios determinados por DRX (polvo total) de diferentes variedades de la Piedra Novelda

(Mineralogy determined by DRX (total powder) of different varieties of the Novelda Stone)

\begin{tabular}{|c|c|c|c|c|c|c|}
\hline \multirow{2}{*}{$\begin{array}{l}\text { VAR IED A D } \\
\text { Variety }\end{array}$} & \multicolumn{4}{|c|}{ B A T E IG } & \multirow{2}{*}{ ALMORQUí } & \multirow[b]{2}{*}{ PORTAZGO } \\
\hline & $\begin{array}{l}\text { B lanca } \\
W \text { hite }\end{array}$ & $\begin{array}{l}A z u \mid \\
B \mid u e\end{array}$ & $\begin{array}{l}\text { Llano } \\
\text { Layer }\end{array}$ & $\begin{array}{l}\text { Fantasia } \\
\text { Fantasy }\end{array}$ & & \\
\hline $\begin{array}{c}\% \text { C A L C ITA } \\
\text { C a lcite }\end{array}$ & $65-70$ & $70-75$ & $65-70$ & $75-80$ & $85-90$ & $75-80$ \\
\hline $\begin{array}{c}\% \text { DOLOMITA } \\
\text { Dolomite }\end{array}$ & $5-10$ & $5-10$ & Traces & Traces & Traces & Traces \\
\hline $\begin{array}{c}\% \text { C U ARZO } \\
\text { Quarz }\end{array}$ & $15-20$ & $10-15$ & $15-25$ & $5-10$ & $5-10$ & $10-15$ \\
\hline $\begin{array}{c}\% \text { FILOSILICATOS } \\
\text { Phyllos ilicates }\end{array}$ & $5-10$ & $5-10$ & $10-15$ & $5-10$ & $5-10$ & $10-15$ \\
\hline
\end{tabular}

TABLA 3/TABLE 3

Parámetros cromáticos of the Novelda Stone

(Chromatic parameters of the Novelda Stone)

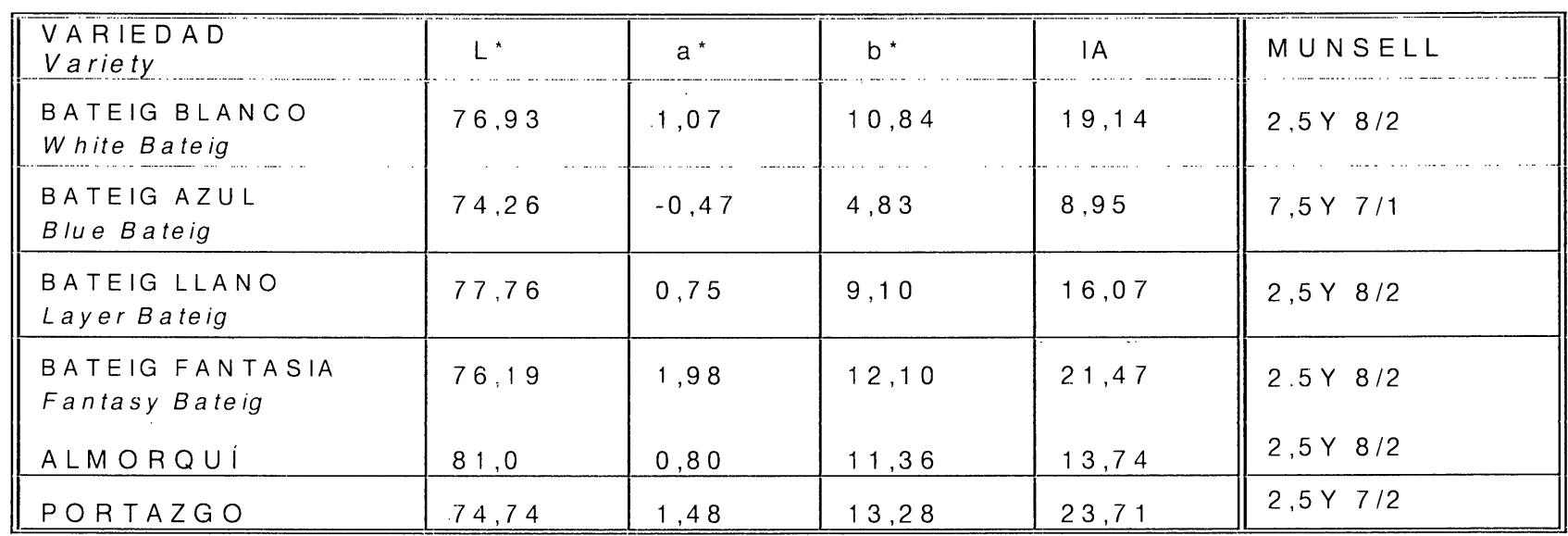


como la nomenclatura Munsell (12). De todas las Piedras de Novelda es la de Almorquí la que tiene una mayor luminosidad ( $\mathrm{L}^{*}$ ), y un índice de amarillo más bajo, junto con el Bateig Azul, mientras que en la caliza de Portazgo el índice de amarillo es el más alto.

La densidad real de las variedades de la Piedra Bateig, Portazgo y Almorquí es del orden de $2.70 \mathrm{~g} / \mathrm{cm}^{3}$. Las densidades aparentes más altas las presentan las variedades Bateig Azul y Blanco, mientras que la Piedra de Almorquí presenta la densidad aparente más baja $\left(2,03 \mathrm{~g} / \mathrm{cm}^{3}\right)$ (Tabla 4$)$.

La velocidad de propagación de las ondas ultrasónicas a través de la Piedra Bateig oscila de los $3.900 \mathrm{~m} / \mathrm{s}$ de las variedades Azul y Blanca a los $3.560 \mathrm{~m} / \mathrm{s}$ de la Piedra Bateig Llano. Las piedras procedentes de las canteras de Almorquí y de Portazgo tienen velocidades ligeramente menores, siendo de $3.445 \mathrm{~m} / \mathrm{s}$ para la primera y $3.335 \mathrm{~m} / \mathrm{s}$ para la segunda (Tabla 4 ).

El Bateig LLano presenta los coeficientes de anisotropía estructural más altos de todas las Piedras de Novelda estudiadas, con un coeficiente $\mathrm{dM}=7,3 \%$. Las medidas de las velocidades de propagación de los ultrasonidos realizadas en las tres direcciones del espacio muestran, para esta variedad, variaciones desde $3.380 \mathrm{~m} / \mathrm{s}$ a $3.680 \mathrm{~m} / \mathrm{s}$. La Piedra de Almorquí presenta una mayor homogeneidad estructural con varieties, Almorqui Stone has the highest lightness ( $L^{*}$ ) and the lowest Yellow index values, alongside Blue Bateig. Portango limestone has the highest values for Yellow Index.

Real density of Porta-go and rarieties of the Bateig is around $2.70 \mathrm{~g} / \mathrm{cm}^{3}$. The highest bulk density values correspond to the Blue and White varieties. Almorqui stone has the lowest bulk density values $\left(2.03 \mathrm{~g} / \mathrm{cm}^{3}\right)$ (Table 4).

Ultrasound propagation velocity through Bateig stone ranges between $3.900 \mathrm{~m} / \mathrm{s}$ obtained for the White and Blue varieties and $3.560 \mathrm{~m} / \mathrm{s}$ for Laver Bateig. Stone from the Almorqui and Portasgo quarries has slightly lower velocity rates $3.435 \mathrm{~m} / \mathrm{s}$ for Almorqui and 3.335 $\mathrm{m} / \mathrm{s}$ for Porta-go (Table 4).

Layer Bateig has the highest structural anisotropy index $(d M=7.3 \%)$. Readings for ultrasound propagation velocity taken in the three directional planes show variations ranging between $3,380 \mathrm{~m} / \mathrm{s}$ and $3,680 \mathrm{~m} / \mathrm{s}$. Almorquí stone is structurally more homogeneous, with values between $3,350 \mathrm{~m} / \mathrm{s}$ and $3.490 \mathrm{~m} / \mathrm{s}$, and it has an anisotropy index $(d M)$ near 4 $\%$, similar to the one that presents the Portasogo Stone.

\section{TABLA 4/TABLE 4}

Propiedades físicas de la Piedra de Novelda

(Physical properties of Novelda Stone)

\begin{tabular}{|c|c|c|c|c|c|c|c|c|}
\hline & \multicolumn{4}{|c|}{ BATEIG } & \multirow[b]{2}{*}{ ALMORQUI } & \multirow[b]{2}{*}{ PORTAZGO } & \\
\hline & & $\begin{array}{l}\text { Blanca } \\
\text { White }\end{array}$ & $\begin{array}{l}\text { Azul } \\
\text { Blue }\end{array}$ & $\begin{array}{l}\text { Llano } \\
\text { Layer }\end{array}$ & $\begin{array}{l}\text { Fantasia } \\
\text { Fantasy }\end{array}$ & & & \\
\hline \multicolumn{2}{|c|}{ Densidad aparente $\left(\mathrm{g} / \mathrm{cm}^{3}\right)$} & 2.26 & 2,29 & 2,13 & 2,19 & 2.03 & 2,16 & Bulk density (g/cm3) \\
\hline \multicolumn{2}{|c|}{ V. Propag. Ultrasonidos (m/s) } & $3.855 \pm 162$ & $3.930 \pm 90$ & $3.560 \pm 135$ & $3.750 \pm 49$ & $3.445 \pm 66$ & $3.335 \pm 123$ & Ultrasonic velocity $(\mathrm{m} / \mathrm{s})$ \\
\hline \multicolumn{2}{|c|}{ Coeficiente anisotropia (dM \%) } & 4,9 & 3,0 & 7.3 & 1,7 & 4,0 & 4,0 & Anisotropy Index (dM \%) \\
\hline \multicolumn{2}{|c|}{ Resistencia compresión ( $\mathrm{MPa}$ ) } & $34,3 \pm 4,4$ & $35.5 \pm 4,1$ & $24,1 \pm 1,7$ & $27,9 \pm 3,6$ & $33,7 \pm 5.6$ & $11,2 \pm 1.8$ & Compressive strength (MPa) \\
\hline \multicolumn{2}{|c|}{ Resistencia a la Flexión (MPa) } & $11,1 \pm 1.3$ & $12,8 \pm 1.4$ & $10,5 \pm 1,7$ & $8.5 \pm 1,8$ & $10.04 \pm 0.5$ & $1,75 \pm 0,37$ & Flexural strength (MPa) \\
\hline \multirow{4}{*}{$\begin{array}{l}\text { Porometria } \\
\text { de } \mathrm{Hg}\end{array}$} & $\%$ Porosidad & 18,7 & 12,7 & 15,9 & 20,4 & 22,3 & 24.2 & Porosity (\%) \\
\hline & $\begin{array}{l}\% \text { Macroporos. } \\
\% \text { Microporos. }\end{array}$ & $\begin{array}{c}0.6 \\
18.1 \\
\end{array}$ & $\begin{array}{c}0,1 \\
12.6 \\
\end{array}$ & $\begin{array}{c}1.1 \\
14,8 \\
\end{array}$ & $\begin{array}{c}0,4 \\
20,0 \\
\end{array}$ & $\begin{array}{r}0,7 \\
21,8 \\
\end{array}$ & $\begin{array}{l}11,6 \\
12,6 \\
\end{array}$ & $\begin{array}{l}\text { Macroporisity (\%) } \\
\text { Microporosity (\%) }\end{array}$ \\
\hline & $\begin{array}{l}\text { T. Medio }(\mu \mathrm{m}) \\
\text { Mediana }(\mu \mathrm{m}) \\
\end{array}$ & $\begin{array}{l}0,05 \\
0.12 \\
\end{array}$ & $\begin{array}{l}0.03 \\
0.08 \\
\end{array}$ & $\begin{array}{l}0.11 \\
0,84 \\
\end{array}$ & $\begin{array}{l}0.08 \\
0.44\end{array}$ & $\begin{array}{l}0.04 \\
0,24\end{array}$ & $\begin{array}{l}0,19 \\
4.59 \\
\end{array}$ & $\begin{array}{l}\text { Mean size }(\mu \mathrm{m}) \\
\text { Mediane }(\mu \mathrm{m})\end{array}$ \\
\hline & Sup. Especif. m2/g) & 6,97 & 7,39 & 2,58 & 4.70 & 12.12 & 2,11 & $\begin{array}{l}\text { Specific surface area } \\
\left(m^{2} / g\right)\end{array}$ \\
\hline \multicolumn{2}{|c|}{$\%$ Saturación } & $7.54 \pm 0.2$ & $6.84 \pm 0.1$ & $10.35 \pm 0.3$ & $8,92 \pm 0.5$ & 10,3 & 9.45 & $\%$ Saturation \\
\hline \multicolumn{2}{|c|}{$\%$ Porosidad accesible al agua } & $17.0 \pm 0.4$ & $15.65 \pm 0.2$ & $21.95 \pm 0,5$ & $19.5 \pm 0,9$ & 20,55 & 20.37 & $\%$ Porosity accesible to water \\
\hline \multicolumn{2}{|c|}{ Coeficiente de absorción } & 5.4 & 5.2 & 7.6 & 6.2 & 6.2 & 6.6 & Coefficient sorption (\%) \\
\hline \multicolumn{2}{|c|}{ Coeficiente de saturación } & 73.0 & 75.5 & 73.6 & 69,6 & 60.0 & 70.0 & Coefficient saturation (\%) \\
\hline \multirow{2}{*}{\multicolumn{2}{|c|}{$\begin{array}{l}\text { Coeficiente capilar }\left(\mathrm{kg} / \mathrm{m}^{2} \mathrm{~h}^{1}{ }^{\prime}\right) \\
\text { Permeabilidad }\left(\mathrm{g} / \mathrm{m}^{\prime} \cdot \mathrm{h}\right)\end{array}$}} & 1.07 & 0.57 & 2,47 & 0.93 & 0.83 & 2.91 & Coefficient of capilar $\left(\mathrm{kg} / \mathrm{m}^{\prime \prime} \mathrm{h}^{\prime} \mathrm{C}^{\prime}\right)$ \\
\hline & & 1.90 & 2.62 & 3.56 & 2,21 & 5.76 & 2.71 & Water vapor permeability $\left(g / m^{\prime} . h\right)$ \\
\hline \multicolumn{2}{|c|}{$\begin{array}{l}\text { Coeficiente permeabilidad } \\
\left(\mathrm{g} / \mathrm{m}^{2} \cdot 24 \mathrm{~h} \mathrm{mmHg}\right)\end{array}$} & 0.33 & 0.38 & 0.55 & 0.39 & 0.38 & 2.24 & Coef. permeability $\left(\mathrm{g}^{\prime} \mathrm{m}^{\prime} \cdot 24 \mathrm{~h} \mathrm{mmHg}\right)$ \\
\hline
\end{tabular}


valores de $3.350 \mathrm{~m} / \mathrm{s}$ y $3.490 \mathrm{~m} / \mathrm{s}$, y un coeficiente de anisotropía $(\mathrm{dM})$ próximo a $4 \%$, similar al que presenta la Piedra de Portazgo. Esta anisotropía es la causa principal de la dispersión que presentan los valores de velocidad de propagación de ultrasonidos

Las variedades que presentan mayor resistencia a la compresión simple corresponden a Bateig Azul y Bateig Blanco, mientras que Bateig Fantasía, Bateig LLano y, sobre todo, la Piedra de Portazgo, tienen los valores más bajos. Igualmente, se puede indicar con respecto a la resistencia a la flexión (Tabla 4). La Piedra de Almorquí tiene una resistencia a la compresión próxima al Bateig Blanco. En función de estos parámetros de resistencia a la rotura la Piedra Bateig y la Piedra de Almorquí pueden catalogarse como de calidad media (13), mientras que la Piedra de Portazgo es de peor calidad mecánica.

\section{COMPORTAMIENTO HÍDRICO}

La degradación de los materiales pétreos está afectada tanto por las condiciones medioambientales en que se van a situar como por las características petrofísicas del material, principalmente por su comportamiento hídrico que vendrá regulado por el sistema poroso (porosidad, distribución de tamaños de poros, forma de los poros, etc). Las calizas de Novelda están caracterizadas por una porosidad alta, entre $12,7 \%$ y $20,4 \%$, en las variedades de la Piedra Bateig. Las piedras de canteras antiguas tienen una mayor porosidad siendo de $22,5 \%$ en las de Almorquí y de $24,2 \%$ en las de Portazgo.

La distribución de tamaños de poros de la Piedra Bateig ha sido estudiada anteriormente (10 y 14). En la Figura 1 se aprecian las diferencias porosimétricas de las Piedras de Novelda. La síntesis porométrica de la Piedra Bateig se muestra en la Tabla 4. La mayoría de los poros se encuentran en el intervalo de microporosidad, $<5 \mu \mathrm{m}$ (14), a excepción de la Piedra de Portazgo que tiene una macroporosidad elevada, alcanzando valores de 11,6\%. Es esta variedad la que tiene un tamaño de poro más alto con una mediana de la distribución de 4,59 $\mu \mathrm{m}$. De la Piedra Bateig es la variedad Bateig Llano la que presenta un tamaño de poro más grande con una mediana de la distribución del 0,84 $\mu \mathrm{m}$. El Bateig Azul es el que tiene un tamaño de poros más pequeños con una mediana de la distribución de $0,08 \mu \mathrm{m}, \mathrm{y}$ un volumen de poros para tamaños inferiores a 0,1 mom del $9,9 \%$ que representa el $78,4 \%$ de la distribución. La superficie específica, medida por el porosímetro de mercurio, es de $12,12 \mathrm{~m}^{2} / \mathrm{g}$ en la Piedra Almorquí, mientras que las más bajas se dan en el Bateig LLano $\left(2,58 \mathrm{~m}^{2} / \mathrm{g}\right) \mathrm{y}$ en la Piedra de Portazgo $\left(2,11 \mathrm{~m}^{2} / \mathrm{g}\right)$.
This anisotropy is the main reason of the dispersion showed in the values of the ultrasound propagation velocity.

White and Blue Bateig are the two varieties with the greatest compressive strength. On the other hand, Fantasy, Layer Bateig and Portasgo stone have the least strength. Results are similar for bending strength tests (Table 4). Almorqui Stone compressive strength is similar to that of White Bateig. Taking into consideration these breakage compressive strength parameters, Blue and White Bateig and Almorqui Stone can be catalogued as medium quality rocks (13), while Porta-go stone ranks as having the poorest mechanical performance.

\section{HYDRIC BEHAVIOUR}

Decay of stone used as building material is directly related to the atmospheric conditions of their location and to the petrophysical characteristics of the stone, mainly its hydric behaviour. This is determined by the porous system of the rock (porosity, pore size distribution, pore shape, etc.). Novelda limestone porosity is high, ranging between $12.7 \%$ and $20.4 \%$ for Bateig stone. Porosity is higher for samples from the Almorqui and Portazgo quarries $122.5 \%$ and $24.2 \%$, respectively).

Pore size distribution of Bateig stone has been previously analysed (10 and 14). Figure 1 shows the porosimetry differences in the various Novelda Stone varieties. Porosimetry results for Bateig stone are summarised in Table 4. The majority of pores fall into the microporosity interval $<5 \mu \mathrm{m}$ (14), except those of the Porta-go stone, with high levels of macroporosity and values of almost 11.6\%. Pores are bigger in this variety, with a median of $4.59 \mu \mathrm{m}$. Of the different types of Bateig stone, Layer Bateig has the biggest pore size, with a median of $0.84 \mu \mathrm{m}$. Blue Bateig pores size is the smallest, with a mean value of $0.08 \mu \mathrm{m}, 9.9 \%$ of volume corresponds to pores under $0.1 \mu \mathrm{m}$, equivalent to $78.4 \%$ of distribution. Specific surface measured by means of the mercury porosimeter is $12.12 \mathrm{~m}^{2} / \mathrm{g}$ for Almorqui Stone. The lowest specific surface readings are obtained for Layer Bateig $\left(2.58 \mathrm{~m}^{2} / \mathrm{g}\right)$ and Portasgo Stone $\left(2.11 \mathrm{~m}^{2} / \mathrm{g}\right)$. 


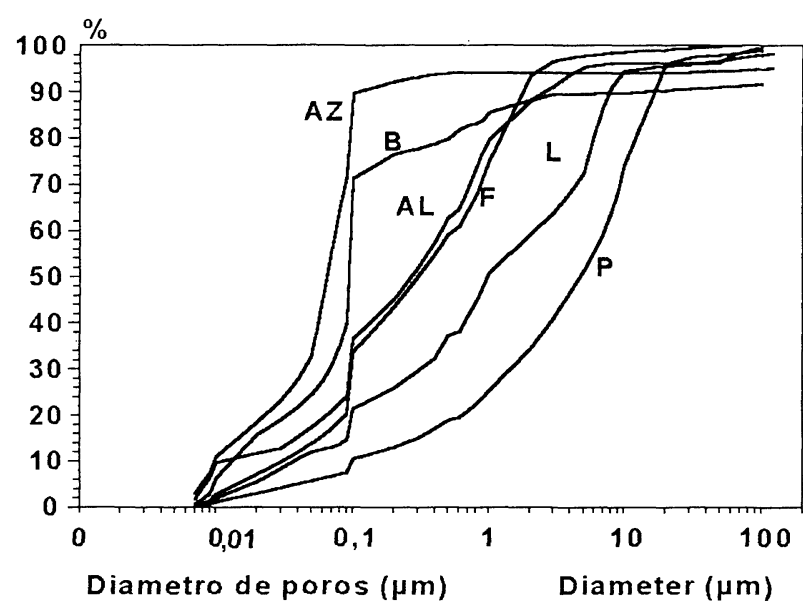

Figura 1.- Diferencias porosimétricas de las Piedras de Novelda.

Figure 1.- Porosimetry differences in the Novelda Stones.

La entrada de agua en la Piedra de Novelda está regulada por este sistema poroso. La cantidad máxima de agua que pueden absorber estos materiales presenta un mayor valor: $10,35 \%$, correspondiente a la variedad Bateig Llano y $9,45 \%$ para la Piedra de Portazgo, y un valor menor: $6,84 \%$, para la variedad Bateig Azul. En la Figura 2 puede apreciarse la absorción de agua por las distintas variedades estudiadas. Son el Bateig Llano y la Piedra de Portazgo, las que, además de absorber mayor cantidad de agua, la absorben más rápidamente, puesto que en la primera hora llegan a succionar cerca del $70 \%$ del total del agua que pueden contener en su interior. La Piedra de Almorquí ticne un comportamiento similar al del Bateig Fantasía, aunque para tiempos largos llega a absorber tanta agua como la procedente de Portazgo. El coeficiente de absorción de agua más alto lo tiene la Piedra Bateig Llano, con un $7,6 \%$, seguida de Portazgo con un 6,6\%. El Bateig Blanco y Bateig Azul presentan los coeficientes de absorción más bajos, $5,4 \%$ y $5,2 \%$ respectivamente.

La Piedra de Portazgo y la Bateig Llano son las que tienen, también, unos coeficientes de capilaridad más altos con valores de $2,91 \mathrm{~kg} / \mathrm{m}^{2} \mathrm{~h}^{1} 2$, para la primera, $y$ de $2,47 \mathrm{~kg} / \mathrm{m}^{2} \mathrm{~h}$ !, para la segunda.La Piedra Bateig Azul tiene una menor capacidad de sorción de agua de lluvia y de agua capilar con un coeficiente de $0,57 \mathrm{~kg} / \mathrm{m}^{2} \mathrm{~h}^{12}$. En la Figura 3 se muestra la cantidad de agua que absorben las distintas Piedras de Novelda por capilaridad.

En la Figura 4 se muestra la cinética de evaporación de agua para estos materiales, con dos etapas claramente diferenciadas. Al comienzo de la evaporación, durante la primera hora. el proceso de secado es más rápido en la variedad de Bateig Azul, evaporándose el 9,3\% del agua que contiene en su interior. En la variedades L Lano y de Portazgo la evaporación es más lenta, no

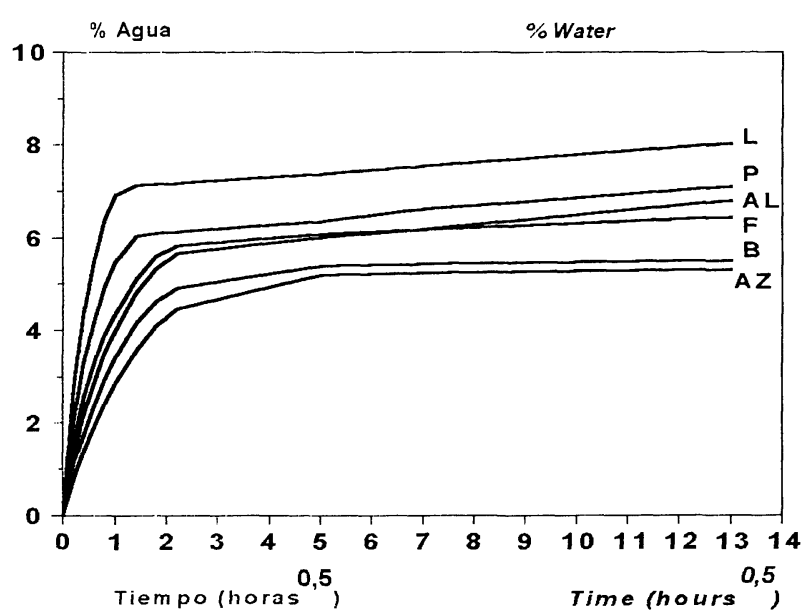

Figura 2.- Absorción de agua de las distintas variedades de piedra estudiadas.

Figure 2. Water absorbed for each stone variets:

The porous system regulates water intake for the Novelda Stone. Maximum water intake ranges between $10.35 \%$, for Layer Bateig and $6.84 \%$ for Blue Bateig. Figure 2 indicates the amount of water absorbed for each stone variety. Portazgo and Layer Bateig absorb more water that other varieties and the kinetic of absorption is faster: They absorb 70\% of the total water which they can absorb during the first hour: Almorqui Stone shows similar behaviour to that of Fantasy Bateig, but when absorbing water over large period of time, it can absorb as much water as the Portazgo Stone. Layer Bateig has the highest absorption index, $7.6 \%$, followed by the Portasgo stone, 6.6\%. White and Blue Bateig have the lowest absorption indexes, $5.4 \%$ and $5.2 \%$ respectively.

Portasgo Stone and Layer Bateig are the two Novelda stone varieties with the highest capillarity indexes, 2.91 $\mathrm{kg} / \mathrm{m}^{2} \quad h^{\prime 2}$ for Portasgo, and $2.47 \mathrm{~kg} / \mathrm{m}^{2} \quad h^{1: 2}$ for Layer Bateig. Blue Bateig can absorb less rainwater and capillary water, with a coefficient of $0.57 \mathrm{~kg} / \mathrm{m}^{2} h^{1.2}$. Figure 3 shows the amount of water absorbed by the different Novelda varieties through capillarity.

Kinetics of water evaporation for these materials is shown in the Figure 4. It can be distinguish two different steps. Drving for the Blue Bateig, at the beginning of the evaporation process, during the first hour, is faster than others: $9.3 \%$ of the water inside the stone is evaporated. Evaporation for Layer variety and Portazgo Stone is slower: Water eliminated is minor 


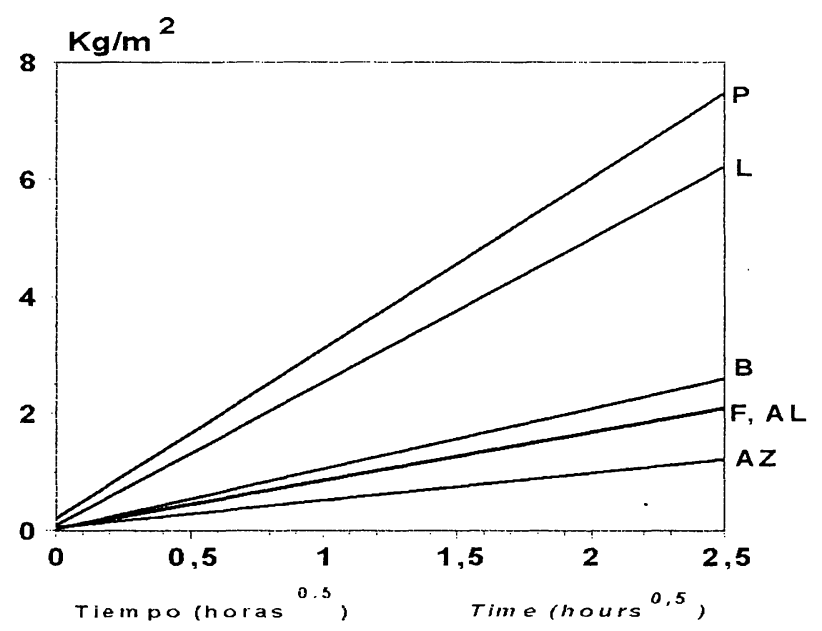

Figura 3.- Cantidad de agua absorbida por capilaridad de las distintas variedades de las Piedras de Novelda.

Figure 3. Amount of water absorbed by the different Novelda Stones rarieties through capillarity:

alcanzando la climinación de agua, en esta primera hora, el $5 \%$ del agua contenida en su interior. $\mathrm{Al}$ finalizar la primera etapa el contenido de agua en el interior de la piedra, (contenido crítico de agua, que marca el punto de inflexión en la curva de velocidad de secado (Figura 4)) (15) es más bajo para las distintas variedades Bateig que para Portazgo y Almorquí. El coeficiente de permeabilidad al vapor de agua, que condiciona la velocidad de secado en la segunda etapa, es muy similar para todas las variedades de Bateig oscilando entre 0,33 y $0,39 \mathrm{~g} / \mathrm{m}^{2} \cdot 24 \mathrm{~h} \cdot \mathrm{mm} \mathrm{Hg}$, a excepción de Bateig Llano que es de $0,55 \mathrm{~g} / \mathrm{m}^{2} \cdot 24$ h.mm Hg. Hay que destacar el valor alto que presenta la piedra de Portazgo del coeficiente de permeabilidad al vapor de agua $2,24 \mathrm{~g} / \mathrm{m}^{2} \cdot 24 \mathrm{~h} \cdot \mathrm{mm} \mathrm{Hg}$, el cual no se refleja en su cinética de secado posiblemente debido a la influencia de los minerales de arcilla que contiene.

\section{DURABILIDAD DE LA PIEDRA DE NOVELDA}

Para la estimación de la durabilidad se han empleado ciclos de simulación de la acción del hielo, de las sales y los cambios humedad-sequedad, pues pueden considerarse los principales mecanismos de deterioro de un material expuesto a unas condiciones externas.

Además es conocido que el sistema poroso, y en concreto la distribución porométrica de una determinada roca condiciona, en gran medida, la accesibilidad de fluidos a su interior y, por tanto, su comportamiento de durabilidad, por lo que se ha utilizado para definir un índice de durabilidad. (1.3, 14, 16 y 17). Según dicho índice la piedra de Almorquí es la que presenta un mejor comportamiento frente al deterioro con índice de durabilidad de 9.1 seguida de la

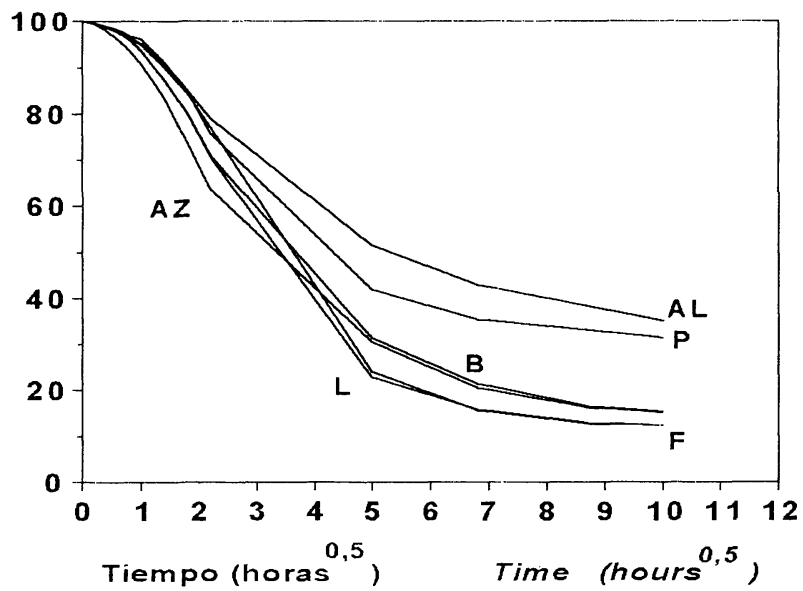

Figura 4.- Cinética de evaporación de agua de los materiales

Figure 4. Kinetics of nater evaporation for these materials.

than $5 \%$ of the total water inside the stone. At the finish of these first step, the water inside the stone (critical water content (15), that show's the inflexion of the dryness kinetic: curve) (Figure 4) for the Bateig varieties is minor than the value for Portasoo and Almorqui stones. Permeability coefficient of water vapour, that conditions drying velocity, is similar for all larieties, ranging from $0.33 \mathrm{to} 0.39 \mathrm{~g} / \mathrm{m}^{2} \cdot 24 \mathrm{~h} \cdot \mathrm{mm} \mathrm{Hg}$. For Layer Bateig permeability coefficient is around $0.55 \mathrm{~g} / \mathrm{m}^{2} \cdot 24 \mathrm{~h} \cdot \mathrm{mm} \cdot \mathrm{Hg}$. Portargo stone shows a permeability coefficient around $2.24 \mathrm{~g} / \mathrm{m}^{2} .24 \mathrm{~h} \cdot \mathrm{mm} \mathrm{Hg}$, this value is not related with its kinetics of drying, it may be for the influence of the clay minerals.

\section{DURABILITY OF NOVELDA STONE}

Durability has been estimated through fieesing/ thaving, wetting/dryness and salt crystallisation tests because these processes are the main mechanisms of damage of the outdoor building materials.

From data of pore size distribution a durability index can be defined (13,14,16 and 17). Almorqui Stone was found to have the highest durability index (9.1). 
Bateig Blanco y Bateig Azul con 7,7 y 7,6

respectivamente. Los peores resultados corresponden a la Piedra de Portazgo $(1,8)$, seguida de la Bateig L Llano y Bateig Fantasía (2.1 y 3,8, respectivamente).

Los resultados obtenidos en ensayos de cristalización de sales (9) indican que, dentro de las variedades de Bateig, es la variedad Bateig Llano la que presenta un peor comportamiento con mayores pérdidas de peso en los diferentes tests, mientras que las otras variedades Bateig tienen un mejor comportamiento frente a la cristalización de sales, con menores pérdidas de peso. Pero los materiales pétreos también están sometidos a otras causas de deterioro como los procesos de hielodeshielo y de humedad sequedad por cambios térmicos. En la Tabla 5 se muestran los \% medios de pérdida de peso de las probetas ensayadas y los valores medios de la velocidad de propagación de ultrasonidos, así como el aumento que experimentan en su capacidad de absorber agua (\% de saturación) después de los ciclos de hielo-deshielo y humedad-sequedad .

El estudio del comportamiento frente a heladicidad realizado en las diferentes variedades de la piedra Bateig muestra un comportamiento similar en cuanto a la perdida de peso, con valores muy alejados del $1 \%$, considerado por la RILEM (5) como significativo de que la roca haya sufrido deterioros importantes. Las variedades Azul, Llano y Fantasía sufren un aumento del agua que pueden contener en su interior (Tabla 5), mientras que el Bateig Blanco no experimenta cambios importantes. La velocidad de propagación de ultrasonidos disminuye significativamente en un $10 \%$ para la variedad Llano, al termino del ensayo de durabilidad, mientras que en la variedad Bateig Blanco su disminución no llega al $2 \%$. La resistencia a followed by. White Bateig and Blue Bateig 7.7 and 7.6. respectively). Worst results are showed by Portazon Stone (1.8), followed by Layer and Fantasy Bateig (2.I and 3.8 , respectively).

The durability of the Bateig stone by means of salt crystallisation tests (9) indicate that the Laver iariety is less durable than other Bateig varieties, according to their behaviour during the tests. Salt crystallisation is not, however, the only factor of decay. There are others. such as water crystallisation and wetting/drying processes induced by temperature changes. Table 5 displays the average percentage values of weight loss in the assayed samples, and average ultrasound propagation values, as well as the increase in water intake capacity (\% saturation) after freesing/thawing and wettingidryness tests.

Bateig varieties show a similar behaviour during the free-ing-thawing tests: Layer and Blue Stones undergo a weight loss percentage during the free-ing-thawing tests, but these values are still far from $1 \%$, the standard value RILEM (5) uses in order to determine significant the decay of the rock. Blue, Layer and Fantasy Bateig water contents increase (Table 5). White Bateig, on the other hand, does not undergo important changes. After the durability test, ultrasound propagation velocity decreases significantly by $10 \%$ in the Layer variety and by under $2 \%$ in the White

TABLA 5/TABLE 5

\% variación en peso, contenido de agua (Ws), velocidad de propagación de ultrasonidos (VPUS) y coficiente de anisotropía $(\mathrm{dM})$, al término de los ensayos de durabilidad en la Piedra Bateig

(\% variation in weight, content of water (Ws), speed of ultrasonic propagation (VPUS) and anisotropy index (aM) at the end of durability test in the Bateig Stone)

\begin{tabular}{|c|c|c|c|c|c|c|c|c|}
\hline \multirow{2}{*}{ BATEIG } & \multicolumn{4}{|c|}{ HIELO-DESHIELO (Freez-Thaw Cycles) } & \multicolumn{4}{|c|}{ HUMEDAD-SEQUEDAD (Humidity-Dryness) } \\
\hline & $\begin{array}{l}\% \Delta \text { Peso } \\
\text { (Weight) }\end{array}$ & $\begin{array}{c}\% \Delta \\
W s\end{array}$ & $\stackrel{\%}{\%} \Delta$ & $\begin{array}{l}\% \Delta \\
\mathrm{dM}\end{array}$ & $\begin{array}{l}\% \Delta \text { Peso } \\
\text { (Weight) }\end{array}$ & $\stackrel{\%}{W} s^{\Delta}$ & $\stackrel{\%}{\text { VPUS }}$ & $\begin{array}{l}\% \Delta \\
d M\end{array}$ \\
\hline $\begin{array}{c}\text { BLANCA } \\
\text { White }\end{array}$ & $-0,06$ & $+0,01$ & $-1,9$ & $+4,6$ & $-0,09$ & $+0,23$ & $-5,0$ & $+37,8$ \\
\hline $\begin{array}{c}\text { AZUL } \\
\text { Blue }\end{array}$ & $-0,10$ & $+0,84$ & $-3,0$ & $+60,6$ & -0.13 & $+1,44$ & $-7,4$ & $+26,4$ \\
\hline $\begin{array}{l}\text { LLANO } \\
\text { Layer }\end{array}$ & $-0,12$ & $+0,58$ & $-9,9$ & $+10,1$ & $-0,12$ & $+0,47$ & $-9,4$ & $+6,0$ \\
\hline $\begin{array}{c}\text { FANTASIA } \\
\text { Fantasy }\end{array}$ & $-0,08$ & $+0,48$ & $-4,4$ & $+59,8$ & $-0,10$ & $+0,71$ & -6.3 & $+28,2$ \\
\hline
\end{tabular}


compresión aumenta después de los ciclos de hielo deshielo (Tabla 5 ). El coeficiente de anisotropía de las probetas sufre un aumento al término del ensayo, tal como se puede apreciar en la Tabla 5.

Es notable que los ciclos hielo-deshielo no perjudiquen la resistencia mecánica de la roca, la cual se mantiene e incluso puede experimentar ligeros aumentos, como viene observándose en diferentes ensayos, algunos de ellos realizados a principios de siglo con motivo de la construcción del edificio de Telefónica de la Gran Vía de Madrid.

Un grado de deterioro ligeramente superior en la Piedra Bateig se produce por las variaciones de humedad-sequedad, tal como se aprecia en los resultados de la tabla 5. Los cuatro tipos de piedra estudiados, después de 20 ciclos de humedad-sequedad, presentan pérdidas de masa similares, próximas al (), 1 \% y un aumento de su capacidad de absorción de agua, alcanzando un incremento de $1,44 \%$ en el Bateig Azul, indicativo de la modificación del sistema poroso. Como consecuencia de la realización de este test se detecta una mayor disminución de la velocidad de propagación de ultrasonidos en todas las variedades Bateig con respecto al ensayo de heladicidad. La Bateig Llano es una excepción al respecto ya que, en ella la disminución de la velocidad de propagación de ultrasonidos es similar en ambos tests.

\section{CONCLUSIONES}

El deterioro que se observa en muchos monumentos construidos con la denominada Piedra de Novelda, principalmente en Monumentos de la Comunidad de Madrid, y con una antigüedad inferior a los cien años, es debido a que se utilizaron canteras con escasa selección de los materiales y de calidad inferior a la que actualmente se está explotando en el Cerro de Bateig, como pudiera ser la Piedra de Portazgo y otras calcarenitas de la zona.

Los índices de durabilidad indican que la Piedra de Portazgo es la que tienc una menor resistencia a los procesos de degradación, mientras que la Piedra de Almorquí, junto con las variedades Bateig Blanco y Azul, tiene la mayor durabilidad. Tal como indicamos la calidad de las distintas variedades de la Piedra Bateig es diferente y su comportamiento en obra estará influenciado por las condiciones medioambientales en que se encuentre el edificio. La utilización de esta piedra como sustitución de piezas deterioradas o para obra nueva tiene que ser seleccionada según parámetros de durabilidad basados en sus características petrofísicas. Las piedras Bateig Azul y Blanco son las que tienen una mejor calidad mecánica y su capacidad de absorción de agua es la más baja de lariety. Compressive strength increase afier freesingl thawing tests (Table 5). Anisotropy index increases by the end of the test, as table 5 reflects.

It is significant that free-ing-thawing (ycles do not have any noticeable effects on the mechanical performance of the stone, which can even improve slightly, as different tests prove. Some of these tests were carried out in the early 1900s on occasion of the building of the Telefónica headquarters in Gran Via (Madrid).

Wetting-dryness process produce greatest decay on Bateig stone, as can be seen from the values in Table 5. The four types of stone analysed undergo similar weight loss after 20 durability wetting-dryness cycles. The veight loss is near $0.1 \%$ and water intake capacity increases by up $101.44 \%$ in Blue Bateig. This fact is related to variation of porous system.

As a consequence on this test, a greater decrease of the ultrasound propagation velocity for all Bateig varieties than in the case of free-ing-thawing tests were detected. For Layer Bateig, this decrease is similar in both tests.

\section{CONCLUSIONS}

The most important decay detected in Novelda Stone in many of the monuments (especially around the Madrid region) in the last century is due to the use of materials from quarries with scarce quality control and inferior quality if compared to that extracted from Cerro de Bateig, for example Portazgo Stone and other calcarenites of the region.

Durability indexes indicate that Portazgo Stone presents the lowest resistance to decay, while Almorqui Stone, and White and Blue Bateig present the best behaviour for durability. As we have already noted, quality of the different Bateig Stone types varies significantly and their performance is directly conditioned by atmospheric conditions around the building. The stone used to substitute decayed piecess or for new buildings must be selected according to durability parameters based on their petrophysical characteristics. White and Blue Bateig stones afford the best mechanical performance results and their water absorption levels are lower than for the rest. Capillary 
todas. La variedad Azul es la que tiene la menor absorción de agua capilar y la Bateig Llano y la Piedra de Portazgo las que tienen una mayor absorción.

La durabilidad de la Piedra Bateig y sus patologías son distintas en las diferentes variedades y frente a los diferentes agentes degradantes considerados. De esta forma, la Piedra Bateig se comporta mejor frente a la heladicidad que frente a las variaciones de humedad sequedad. La variedad Bateig Blanco es la que mejor resiste los ciclos de hielo-deshielo y cristalización de sales, siendo relativamente la que presenta una mayor durabilidad.

Las variedades Azul y Fantasía no presentan una mala durabilidad, si bien se detectan cambios en su anisotropía como resultado de los ciclos de hiclodeshielo. La variedad Llano es la variedad de Bateig que presenta una menor durabilidad si bien en clla los procesos de hielo-deshielo y humedad sequedad no aumentan su anisotropía, si no que tienden a provocar una arenización homogénea.

La Piedra Bateig es más sensible a las variaciones de humedad-sequedad, ya que todas las variedades aumentan su grado de anisotropía Únicamente en estas condiciones es la variedad Bateig Llano la que tiene un comportamiento diferente, con escaso incremento de su anisotropía e inicios de procesos de arenización.

Atendiendo al índice de anisotropía, se puede predecir que, después de muchos ciclos de tests, las Piedras Bateig Blanco y Azul tenderán a sufrir procesos de descamación y fisuraciones paralelas a la superficie de los sillares o placas de piedra, mientras que el Bateig Llano tenderá a sufrir arenización

Por último indicar que la falta de selección y control de calidad del material puesto en obra y la utilización conjunta de las distintas variedades en un mismo paramento pueden acelerar el proceso de degradación de algunos de los sillares, dadas las diferentes propiedades petrofísicas de los materiales, que los pueden hacer incompatibles entre sí.

Para mejorar su comportamiento en obra sería aconsejable utilizar tratamientos de protección (18 y 19), previos a su colocación. En el caso de los aplacados se deberían colocar con técnicas de sujeción especiales que ayuden a aislar a la piedra de los efectos del agua.

\section{AGRADECIMIENTOS}

Este trabajo ha sido financiado por DGES (proyecto PB 96-0321) y la Comunidad de Madrid (proyecto 05P(045/1996). Queremos agradecer las facilidades dadas por Bateig, S. A. water sorption is lower for Blue Bateig. On the other hand, Layer Bateig and Porta-go Stone obtain the worst results.

Bateig Stone durability and its pathologies differ depending on the decay agents considered. Thus, durability when faced with free-e-thaw is higher than when faced with humidity variations. White Bateig is the most resistant to freeze-thaw and salt crystallisation cycles, it is the Bateig variety with the greatest durability.

Blue and Fantasy varieties do not show a bad durability, although changes in the anisotropy are generated by the free-ing-thawing tests in this stones. Layer variety is the Bateig variety with the least durability although the freesing/thawing and wetting/ dryness cycles do not increase its anisotropy, otherwise these processes cause the granular disintegration.

Bateig Stone is more sensitive to humidity variations, since all varieties undergo an increase in anisotropy and a decrease in ultrasound propagation velocity. Performance of Layer Bateig does not decrease significantly under these conditions, as its anisotropy remains quite stable and indices of granular disintegration can appear.

From anisotropy index, it can be predict scaling and fissuring after a great number of cycles of the different durability test on White and Blue Bateig stones, while Layer Bateig tends to undergo sanding processes.

Finally, we should note that the lack of attention to selection processes and quality control in the choice of materials, as well as the combined use of different varieties can accelerate the decay process on account of the different petrophysical properties of the varieties that rend them incompatible.

In order to improve performance it would be advisable to apply protective treatments prior to use (18 and 19). In the case of the stone slabs it would be advisable to apply special support techniques that have a waterproofing effect on the stone.

\section{ACKNOWLEDGEMENTS}

This study was supported by DGES Research Project PB 96-0.32I and Comunidad of Madrid Project 05P-(0451 1996. We thank Bateig, S. A.. for their assistance. 


\section{BIBLIOGRAFÍA}

(1) S. Ordóñez. M.A. García del Cura. A. Bernabeú y M.A. Rodríguez: Rocas ornamentales porosas del Mioceno marino de Levante (Alicante-Murcia-Albacete), En “Avances en el conocimiento del Terciario Ibérico" J.P.Calvo y J.Morales (eds). 141-144. UCM. Madrid. 1997.

(2) M. Louis. J. Alonso. V. Martínez y J.S. Alcaide: Caratéristiques du grès natural de Bateig très utilisé dans L architecture. 7 th. Int. Cong. on Deterioration and Conservation of Stone. 1205-1212. Lisboa. 1992.

(3) J.M. García de Miguel. L. Sánchez. O. Puche y M.T. González $\Lambda$ guado: Study of the monumental stone from Madrid district. $7^{\text {th }}$ Int. Cong. On Deterioration and Conservation of Stone. 47-56. Lisboa, 1992.

(4) J.M. García de Miguel. L. Sánchez y M.T. González Aguado: Characterization and deterioration causes of the Novelda Stone. III Int. Sym. on the Conservation of Monuments in the Mediterranean Basin. 547-554. Venecia 1994.

(5) UNE: Mármoles y calizas ornamentales: Resistencia a la compresión (UNE 22-185-85). Resistencia a la flexión (UNE 22-17685) y Resistencia a las heladas (UNE 22-184-85). Instituto Español de Normalización. Madrid. 1985.

(6) RILEM: Recommended test to measure the deterioration of stone and to asses the effectiveness of treatment methods. Commission 25-PEN: Protection et erosion des Monuments (1980), pp. 175-253.

(7) U. Zezza: Physical-mechanical properties of quarry and building stones. Analytical Methodologies for investigation damages stones, pp 20. Pavia (Italia), 1990.

(8) CNR-ICR: Normal 43/93 Misure colorimetriche strumentali di superfici opache. Italia, 1994.

(9) D. Benavente, M.A. García del Cura, A. Bernabéu. A. y S. Ordóñez:

Quantification of salt weathering in porous stones using an experimental continuous partial immersion method. Engineering Geology. 59, 3-4 (2001) pp. 315-327.

(10) S. Ordóñez. M.A.. García del Cura, R. Fort. M. Louis, M.C. López de Azcona y M. Mingarro: Physical properties and petrographic characteristics of some Bateig stone varietics. En: 7th Int. Cong. International Association of Engineering Geology (ed. R.Oliveira. L.F. Rodrigues. A.G. Coelho \& A. P. Cunha). pp. 3595-3603. Rotterdam. 1994.

(11) A. La Iglesia, M.A. García del Cura. S. Ordóñez. y A. Bernabéu: Estudio de los fïlosilicatos de la "Piedra Bateig̣" (Neógeno do la Provincia de Alicante). Geogaceta. 23. (1998), pp. 79-82.

(12) MUNSELLL COLOR. Munsell Soil Color Charts. Baltimore. Maryland. 1977.

(1.3) ASTM: Anual Book of ASTM Standards, part. 19. Soil and Rock: Building Stones C568-79. 1981

(14) S. Ordóñez. R. Fort, M.A. y García del Cura: Pore size distribution and the durability of a porous limestone. The Quarterly Journal of Engineering Geology. 30 (1997). pp. 221-2.30.

(15) R. Fort. El agua en los materiales pétreos: comportamiento hídrico. En "Degradación y conservación del Patrimonio Arquitectónico”. Ed. F. Mingarro. pp 237-248. Ed.Complutense. Madrid. 1996.

(16) S.A. Russel: Stone preservation committee report H.M. Stationary Offïce. London. 1927.

(17) D. Benavente. M.A. García del Cura. R. Fort y S. Ordóñez: Thermodynamic modelling of changes induced by salt pressure crystallisation in porous media of stone. Journal of Crystal Growth. 204 (1999). pp.168-178.

(18) A. Bernabéu. Mejora de calidad de materiales pétreos porosos mediante cementación inducida. Tesis Doctoral (en preparación).

(19) A. Bernabeú. E. Expósito, V. . S. Ordóñez. \& A. Aldaz: A new electrochemical method for consolidation of porous rocks. Electrochemistry Communications $\left.3 . n^{\prime \prime} 3(20) 1\right)$ pp. 122-127 